\title{
X-ray Testing at PANTER of Optics for the ATHENA: and Arcus Missions
}

\author{
Vadim Burwitz \\ Max-Planck-Institut für extraterrestrische Physik \\ October 10, 2018 ICSO Conference \\ Chania, Crete, Greece
}




\section{Overview}

- The ATHENA:and ARCUS missions

- The PANTER X-ray test facility

- Optics development, and AIT tests at PANTER

- Preparing the Athena Calibration

- Test-, Full Illumination-, Sectorwise measurements

- Facility upgrades

- Summary 


\section{Athena (ESA) L-Mission}

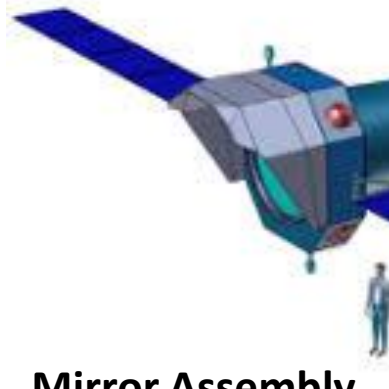

Mirror Assembly

1000 Silicon Pore Optic Mirror Modules

Diameter $\quad 0.5 \mathrm{~m}-2.5(3.0) \mathrm{m}$

Focal Length $12 \mathrm{~m}$

Weight $\quad<1000 \mathrm{~kg}$

On-axis spatial resolution

HEW (50\% encircled energy) :

- 5 arcsec

- $290 \mu \mathrm{m}$

Collecting area at $1 \mathrm{keV}$

$14500 \mathrm{~cm}^{2}(20000) \mathrm{cm}^{2}$ coated silicon plates

Planned Adoption 2021

\section{Arcus (NASA) MIDEX}

Arcus Optical Layout

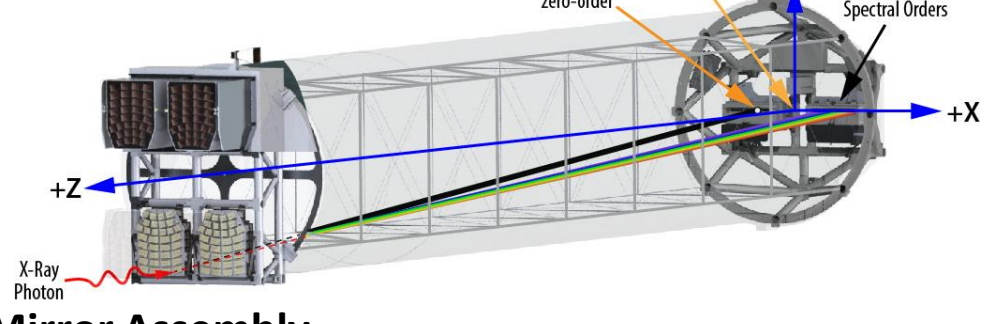

Mirror Assembly

4 Petals each with 34 Silicon Pore Optic Mirror Modules

Radii

$0.35 m-0.8 m$

Focal Length $12 \mathrm{~m}$

On-axis spatial resolution

HEW (50\% encircled energy) :

- 5 arcsec or better

- $290 \mu \mathrm{m}$

- Transverse PSF < 2“ $\left(1.5^{\prime \prime}\right)$

$\rightarrow$ drives spectral resolution $>2500$

Collecting area at $1 \mathrm{keV}$

$>450 \mathrm{~cm}^{2}$ coated silicon plates

Transition from Phase A to Phase B

$\rightarrow$ pending ongoing review 


\section{The MPE PANTER X-Ray Test Facility}

- Located in Neuried, south west of Munich

- $120 \mathrm{~m} \mathrm{X}$-ray beamline, $1 \mathrm{~m}$ diameter

$\rightarrow$ beam divergent
- Large cleanroom for handling X-ray optics

- Movable $10 \mathrm{~m}$ extension with $0.25 \mathrm{~m}$ diameter and $3 \mathrm{~m}$ instrument chamber, $1.2 \mathrm{~m}$ diameter

- $12 \mathrm{~m}$ instrument chamber, $3.5 \mathrm{~m}$ diameter

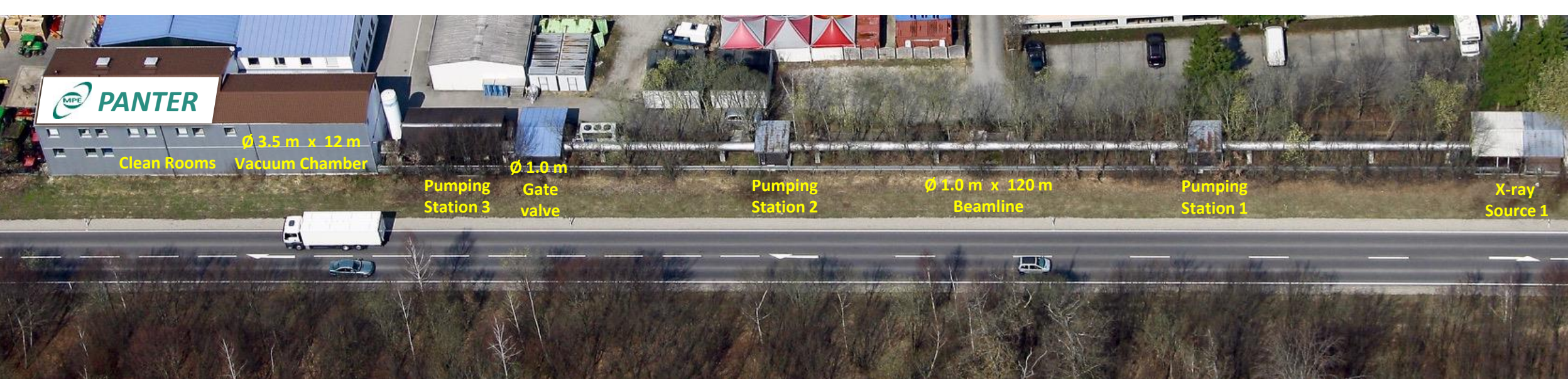




\section{The MPE PANTER X-Ray Test Facility}

- Located in Neuried, south west of Munich

- $120 \mathrm{~m}$ X-ray beamline, $1 \mathrm{~m}$ diameter

- $12 \mathrm{~m}$ instrument chamber, $3.5 \mathrm{~m}$ diameter
- Large cleanroom for handling X-ray optics

- Movable $10 \mathrm{~m}$ extension with $0.25 \mathrm{~m}$ diameter and $3 \mathrm{~m}$ instrument chamber, $1.2 \mathrm{~m}$ diameter

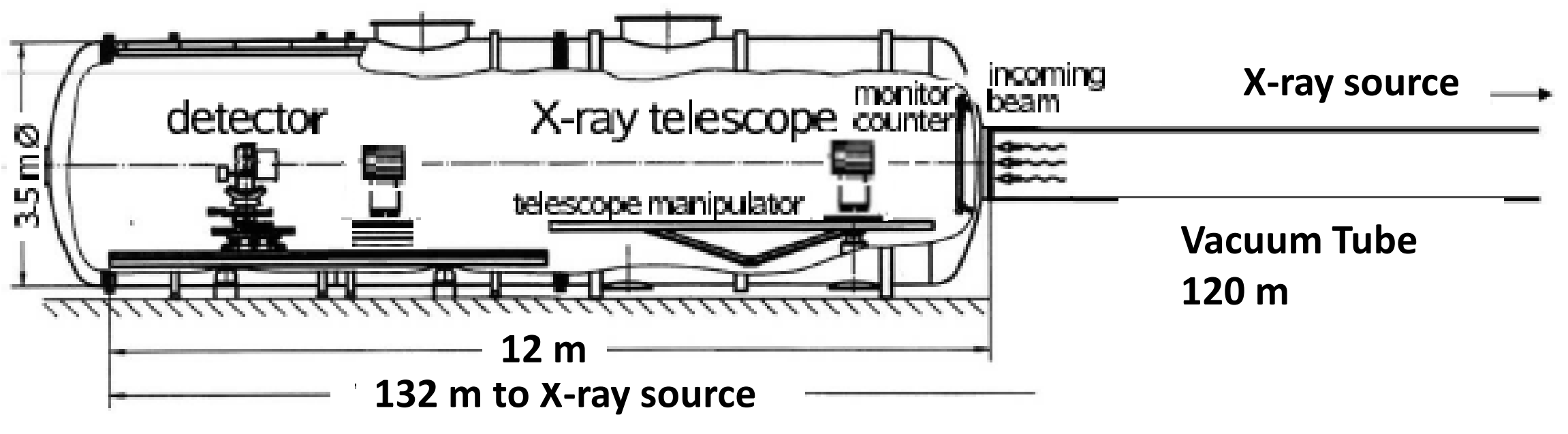




\section{Upgrades to PANTER to measure ATHENA: Mirror Modules}

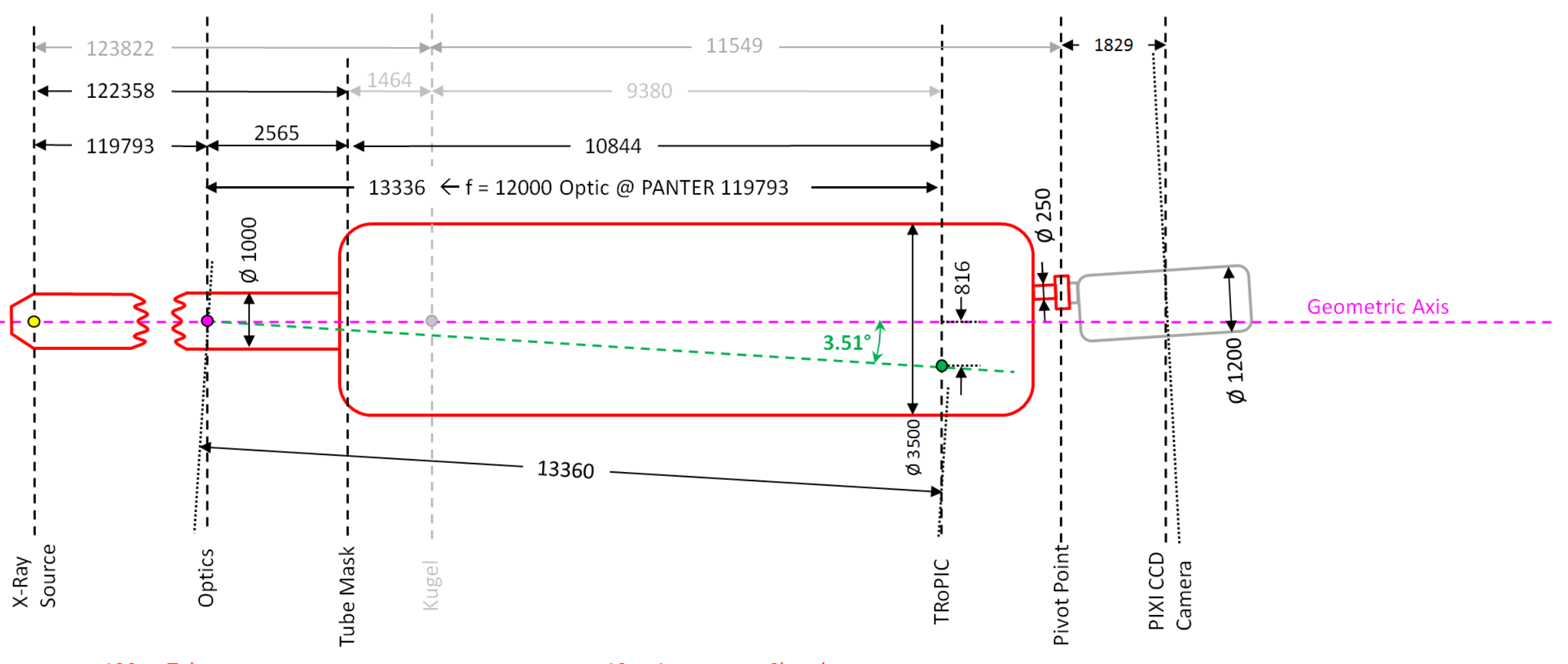




\section{PANTER Upgrades for ATHENA: and ARCUS}

- New thermally controlled optical bench in 1-m diameter Tube

- TRoPIC and PIXI camera Manipulator upgraded to allow $1.2 \mathrm{~m}$ vertical, $1.2 \mathrm{~m}$ horizontal, and $0.54 \mathrm{~m}$ focus movements for in the chamber.

$\rightarrow$ to accomodate large radius Athena SPO optics, as well as ARCUS gratings

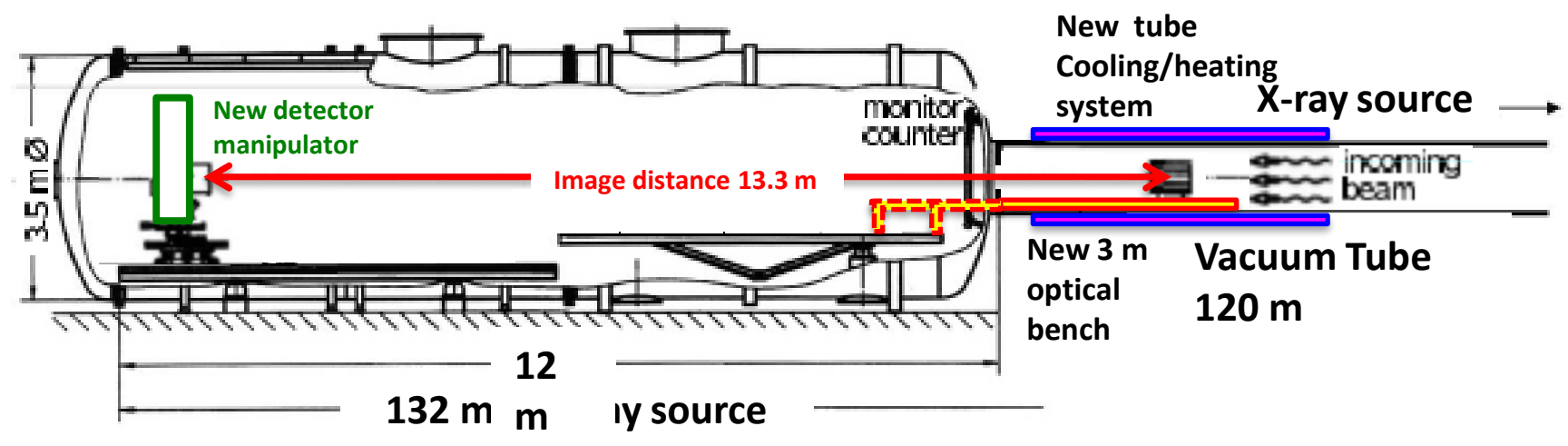




\section{PANTER Upgrades for ATHENA: and ARCUS}
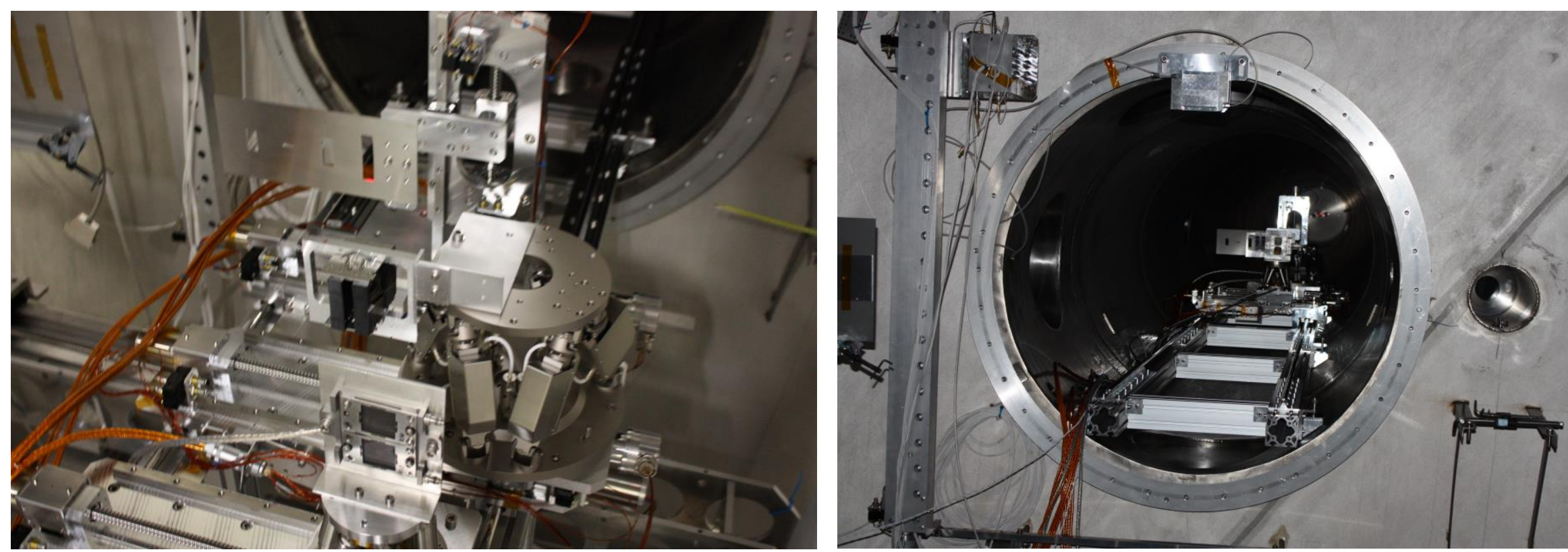


\section{PANTER Upgrades for ATHENA:and ARCUS}
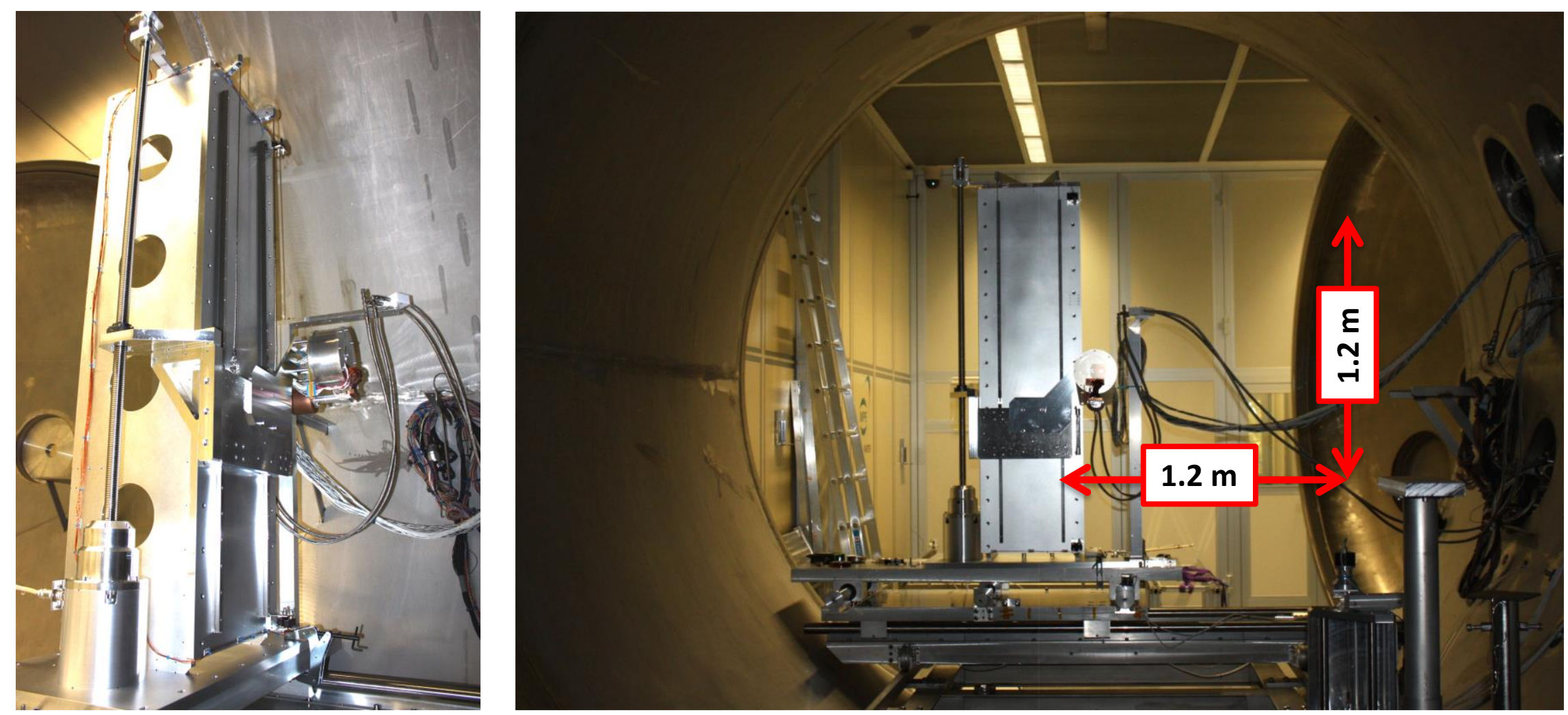


\section{PANTER Upgrades for ATHENA: and ARCUS}

- New thermally controlled optical bench in 1-m diameter Tube

- TRoPIC and PIXI camera Manipulator upgraded to allow $1.2 \mathrm{~m}$ vertical, $1.2 \mathrm{~m}$ horizontal, and $0.54 \mathrm{~m}$ focus movements for in the chamber.

$\rightarrow$ to accomodate large radius Athena SPO optics, as well as ARCUS gratings

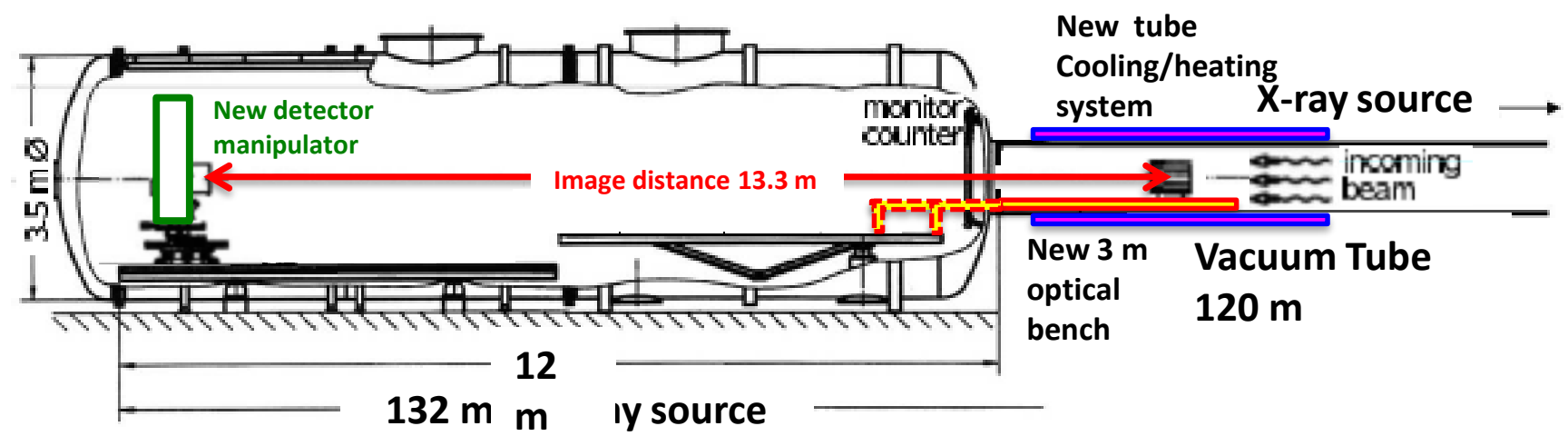




\section{Silicon Pore Optics Technology}

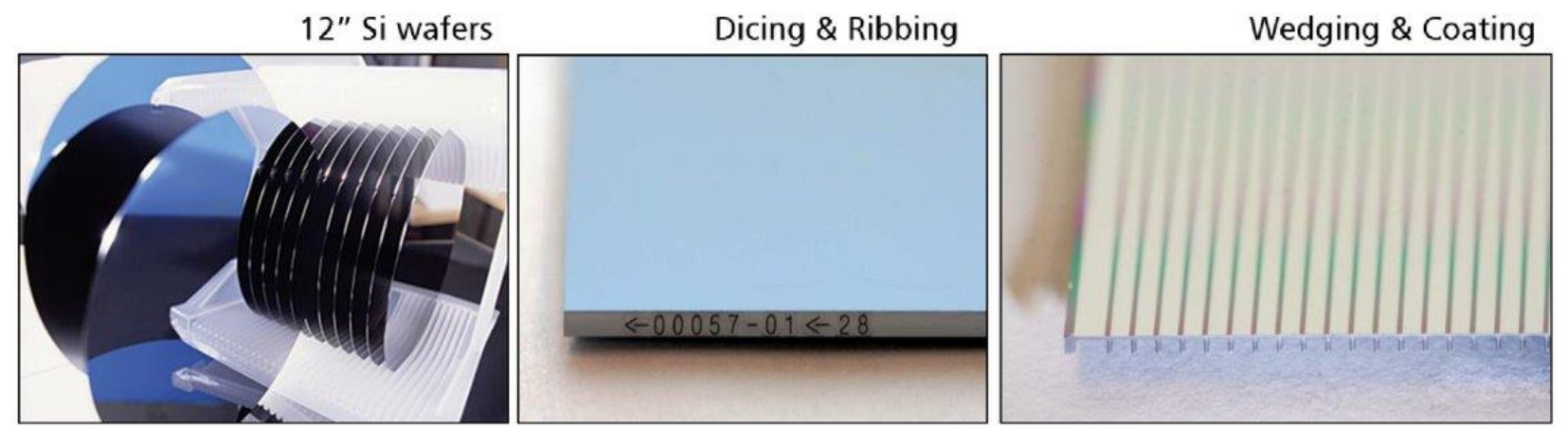

Was discussed In detail in the previous talk by Max Collon

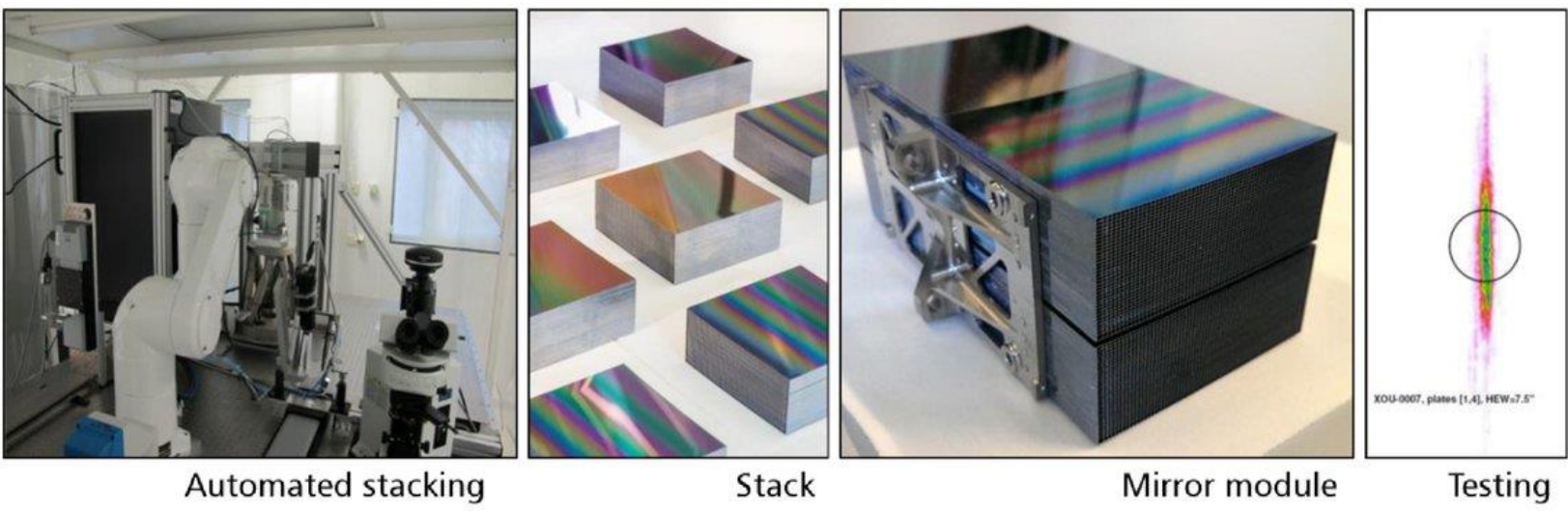




\section{The Baseline ATHENA:OOptics}

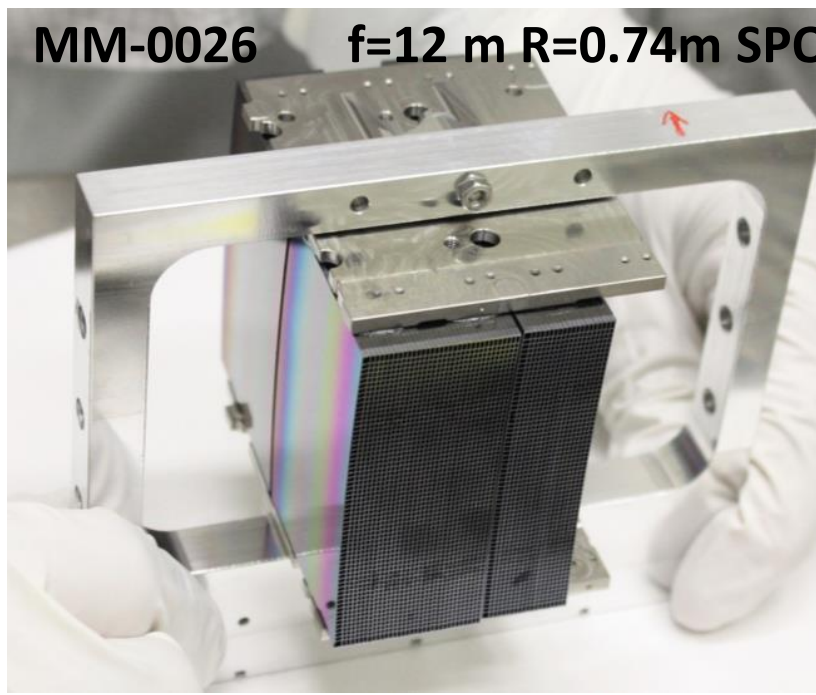

middle radius

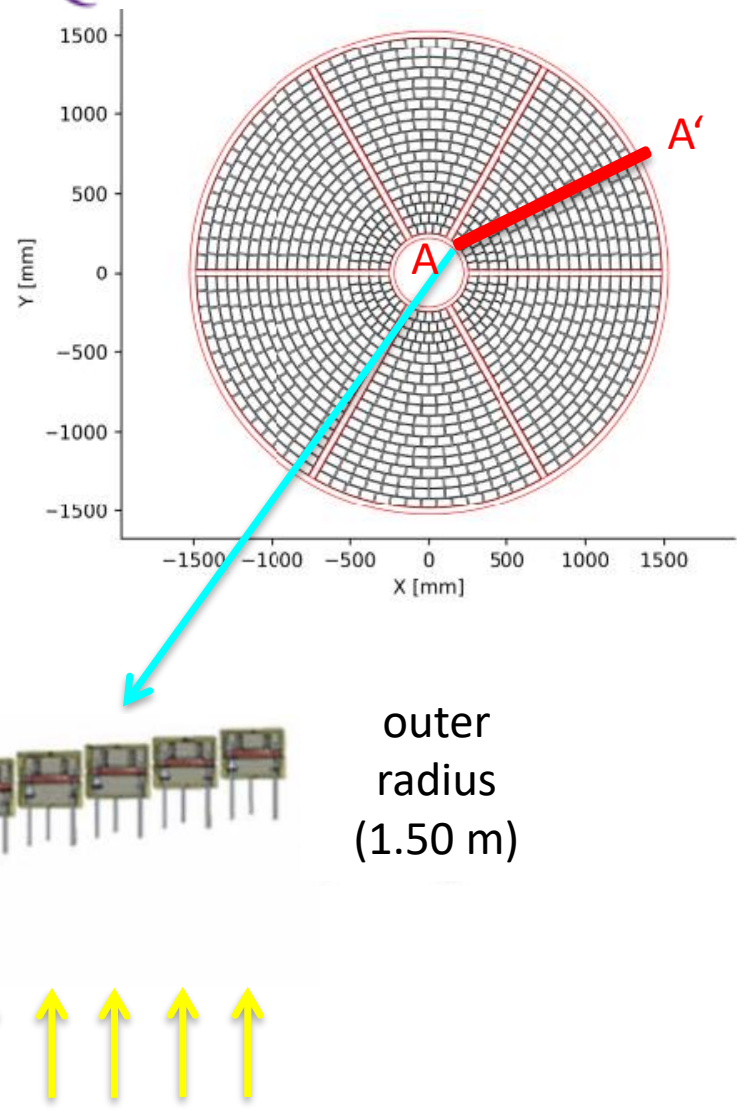

$0.74 \mathrm{~m}$

$\downarrow A-A^{\prime}$

radius

$0.24 \mathrm{~m}$

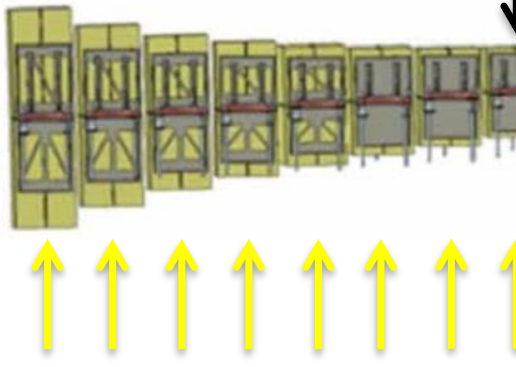

$$
\text { X-rays }
$$




\section{The Baseline Arcus Optics}

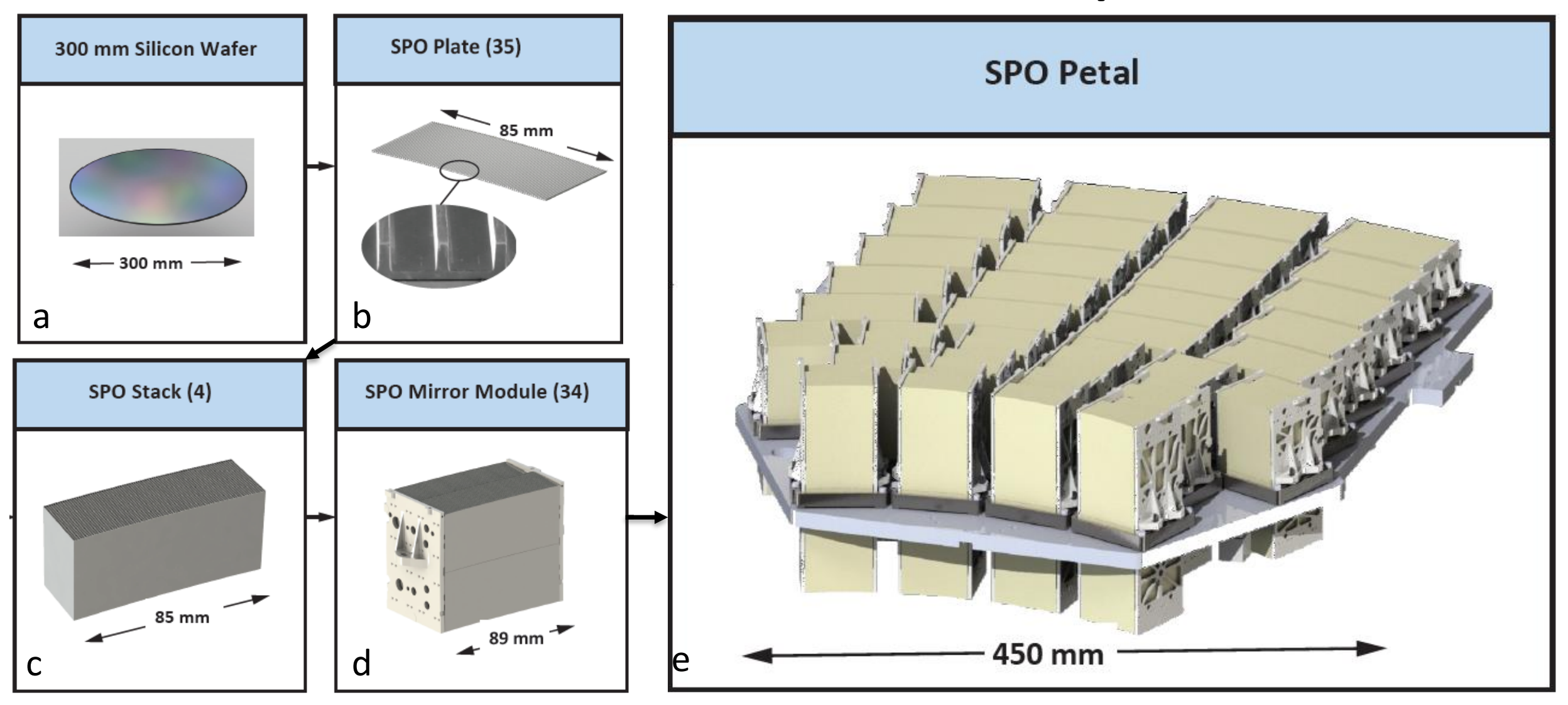

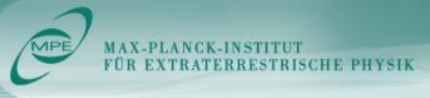




\section{Testing X-ray Telescope optics}

Optical Tests in

Production Laboratories

- Profilometers

- Null lenses

- Roughness

- Reflectometers
$X$-ray Test Facilities

- BESSY

- Pencil beam scanning

- Used during developement and mirror module production

- Long Beam Facility (PANTER)

- Full illumination of completed mirror modules 


\section{Testing X-ray Telescope optics at PANTER}

Test sequence

- Alignment using Laser on Air

- X-ray Alignment

- Pitch

- Yaw

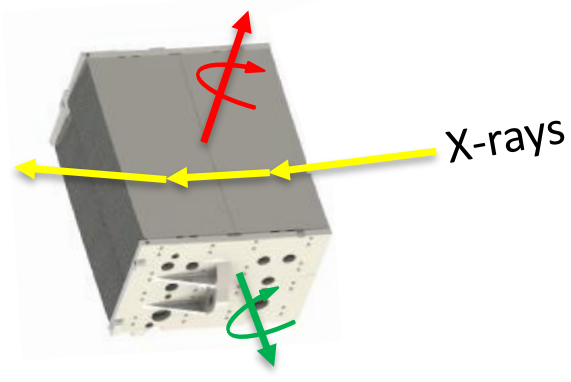

Measurements at several energies

- PSF

- On-axis

- Off-Axis

- Hartmann Test

- Intra / extra focal images

- $\quad$ Effective area

- On-axis

- Off-Axis

- Focus Search 
SPO Tests at PANTER: Pitch \& Yaw
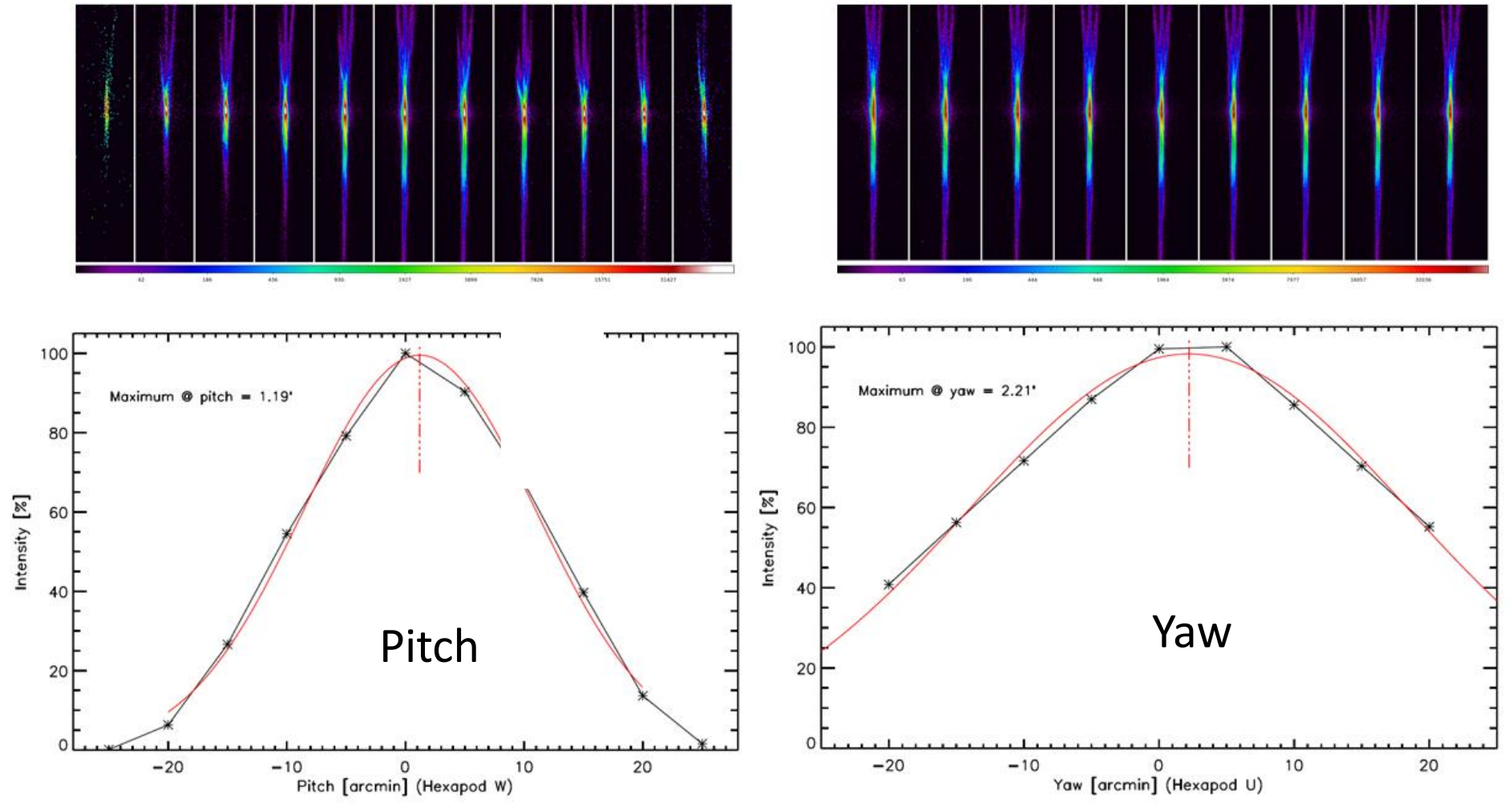


\section{SPO Tests at PANTER: Focus Search}
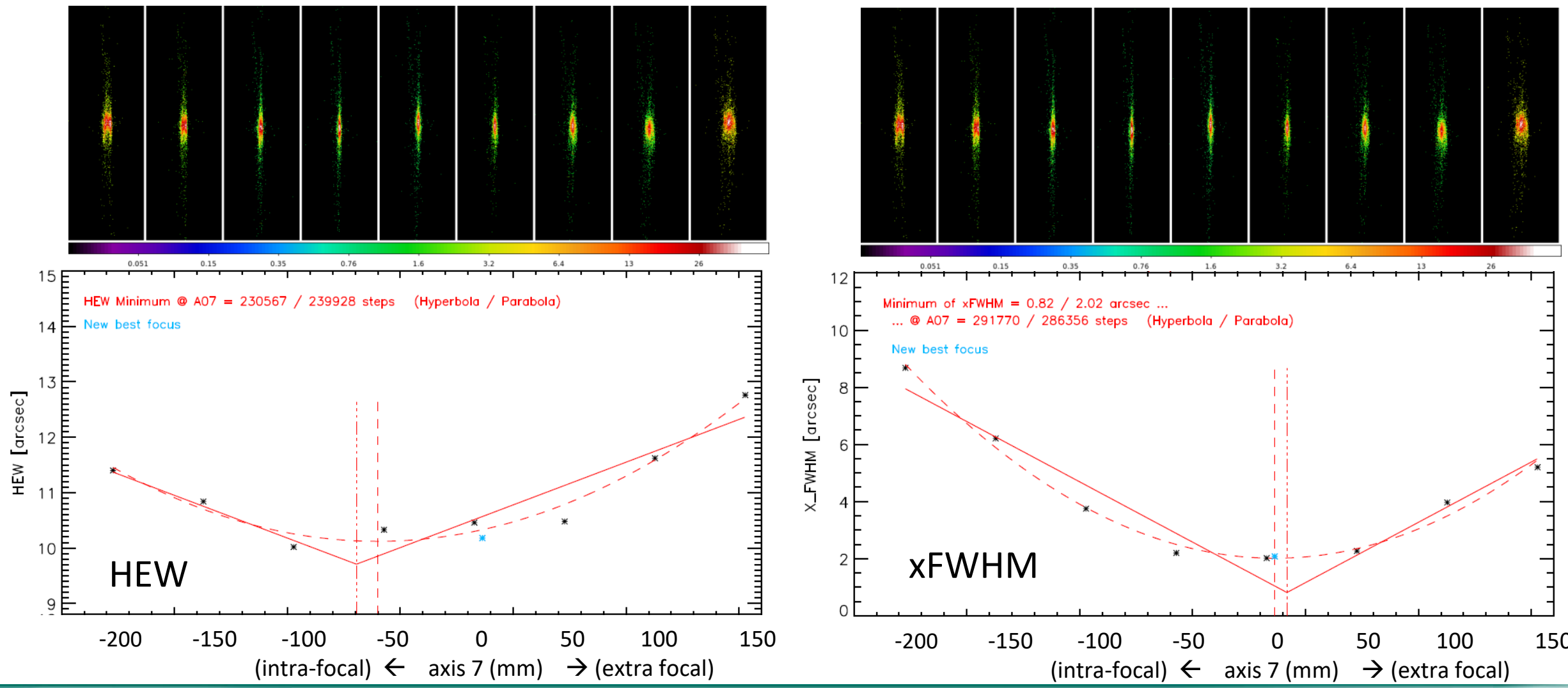


\section{SPO: PIXI azimuthal (Hartmann) scan}

$\leftarrow$ Wald

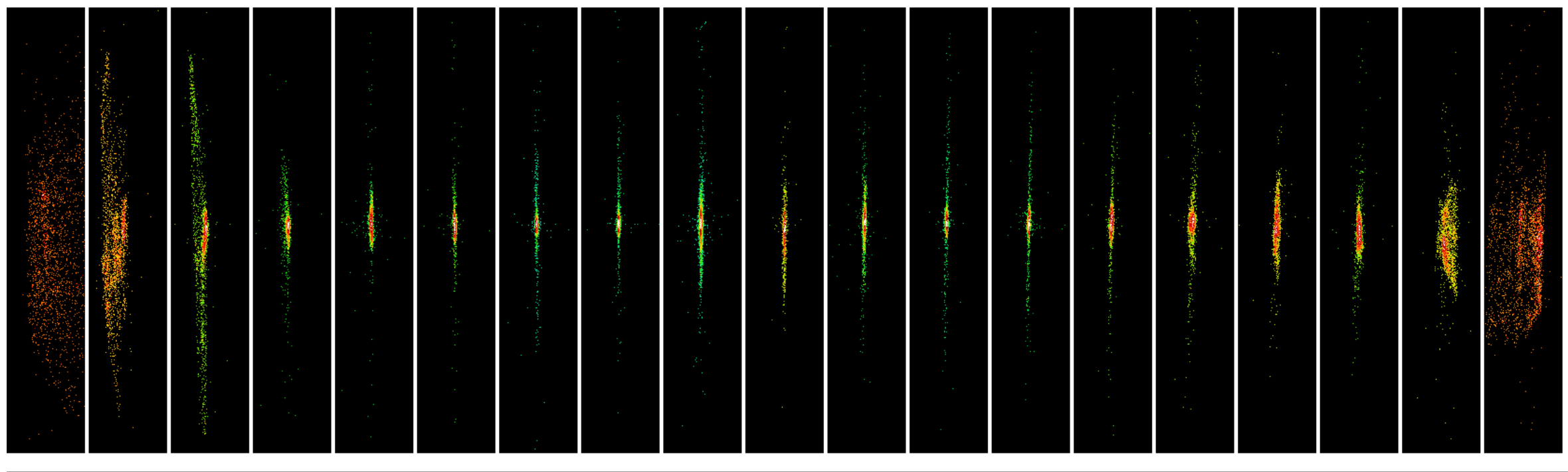

Pantolsky $\rightarrow$ 


\section{Testing unglued SPO optics in PANTER}

Two test stacks $1 \mathrm{~mm}$ separation

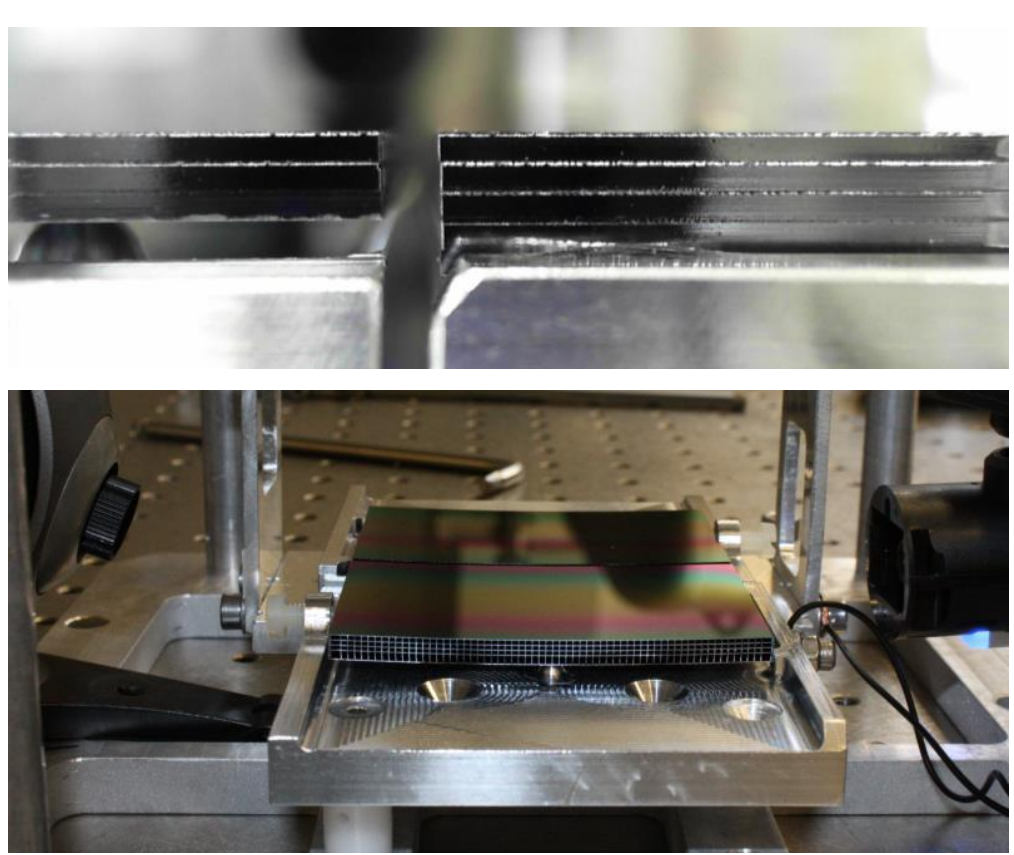

Setup prior to shifting to final position

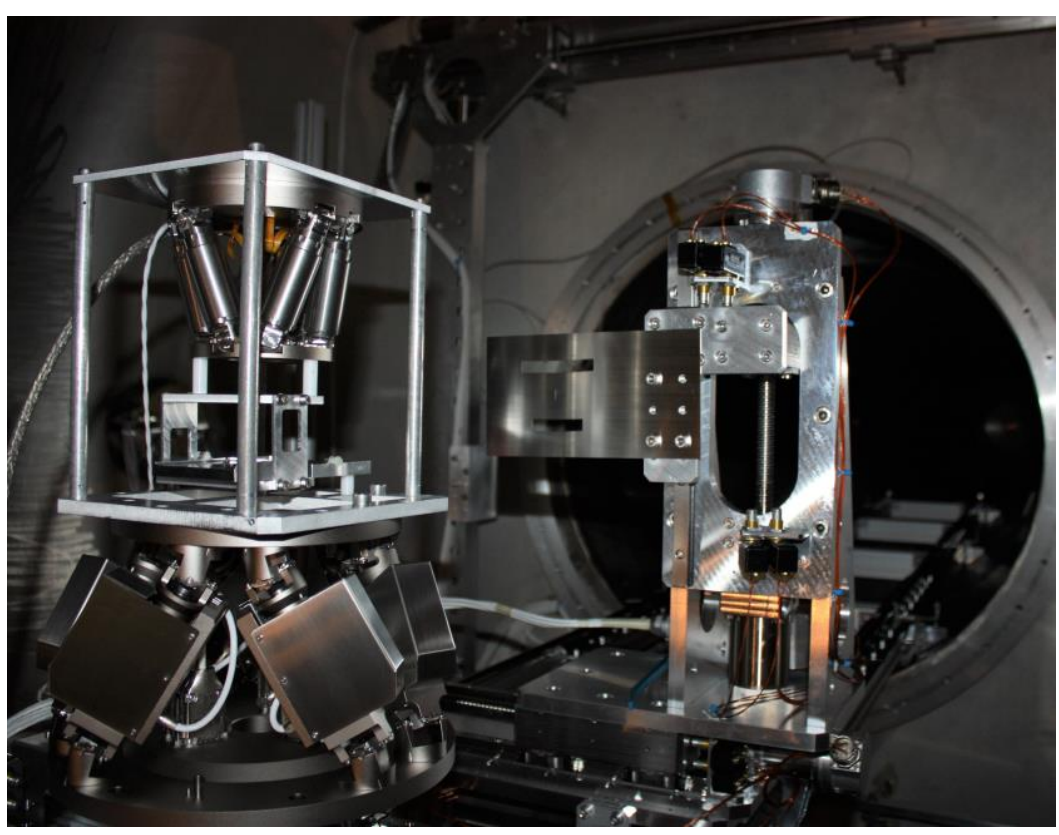




\section{Testing unglued SPO optics in PANTER}

XOU-0060C Mirror scan of direct beam through primary and secondary optic

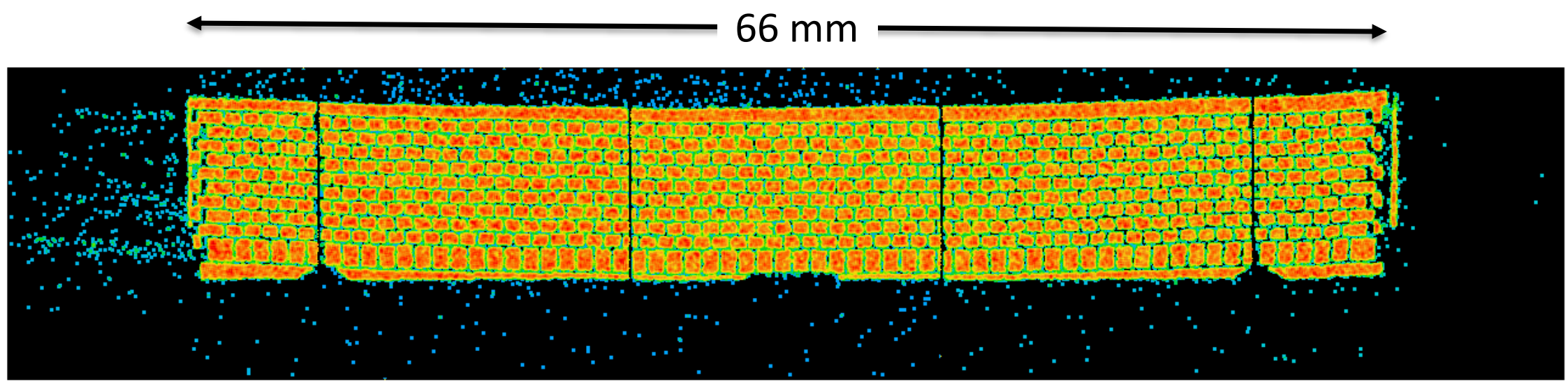




\section{Testing X-ray Telescope Petals at PANTER}

Media Lario ESA ATHENA Mirror AIT

UV Alignment Technique (Talk this morning ICSO, G. Valsecchi )

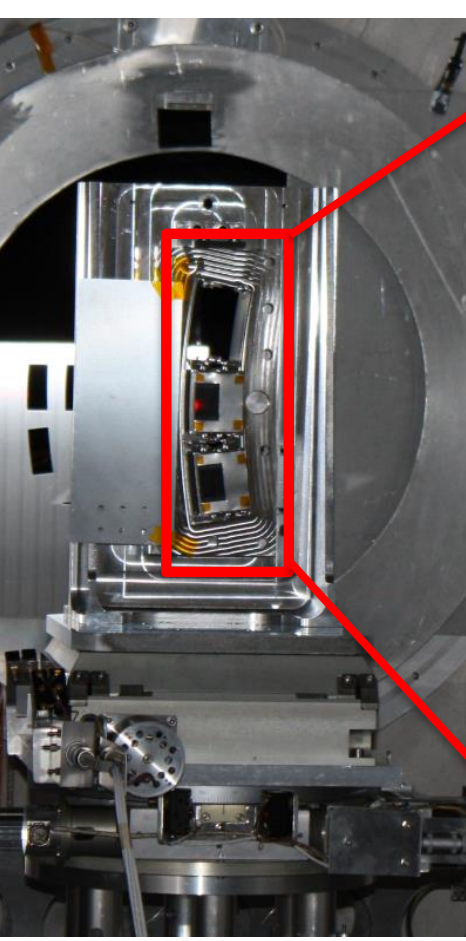

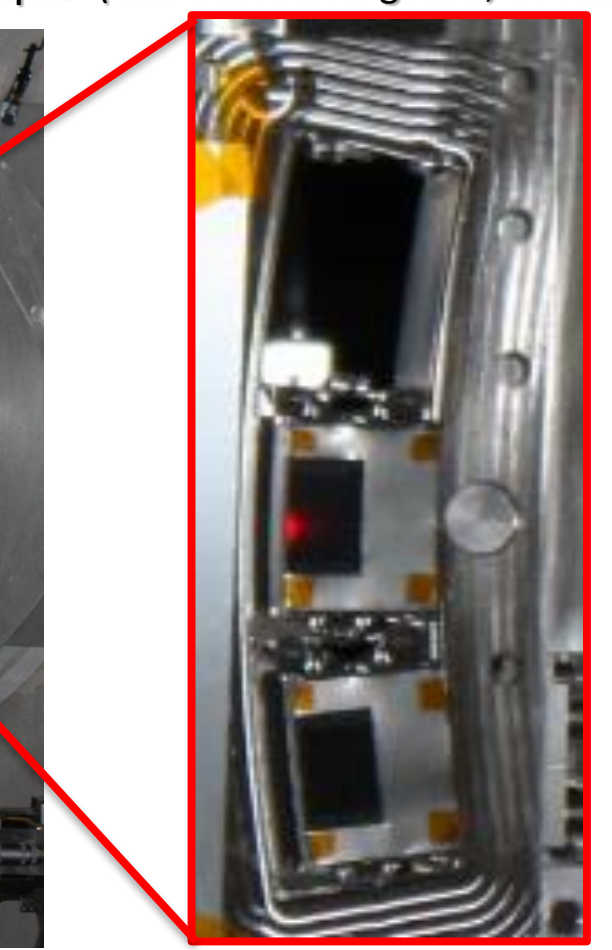

RUAG ESA ATHENA Mirror AIT

Alignment cube metrology \& X-ray alignment

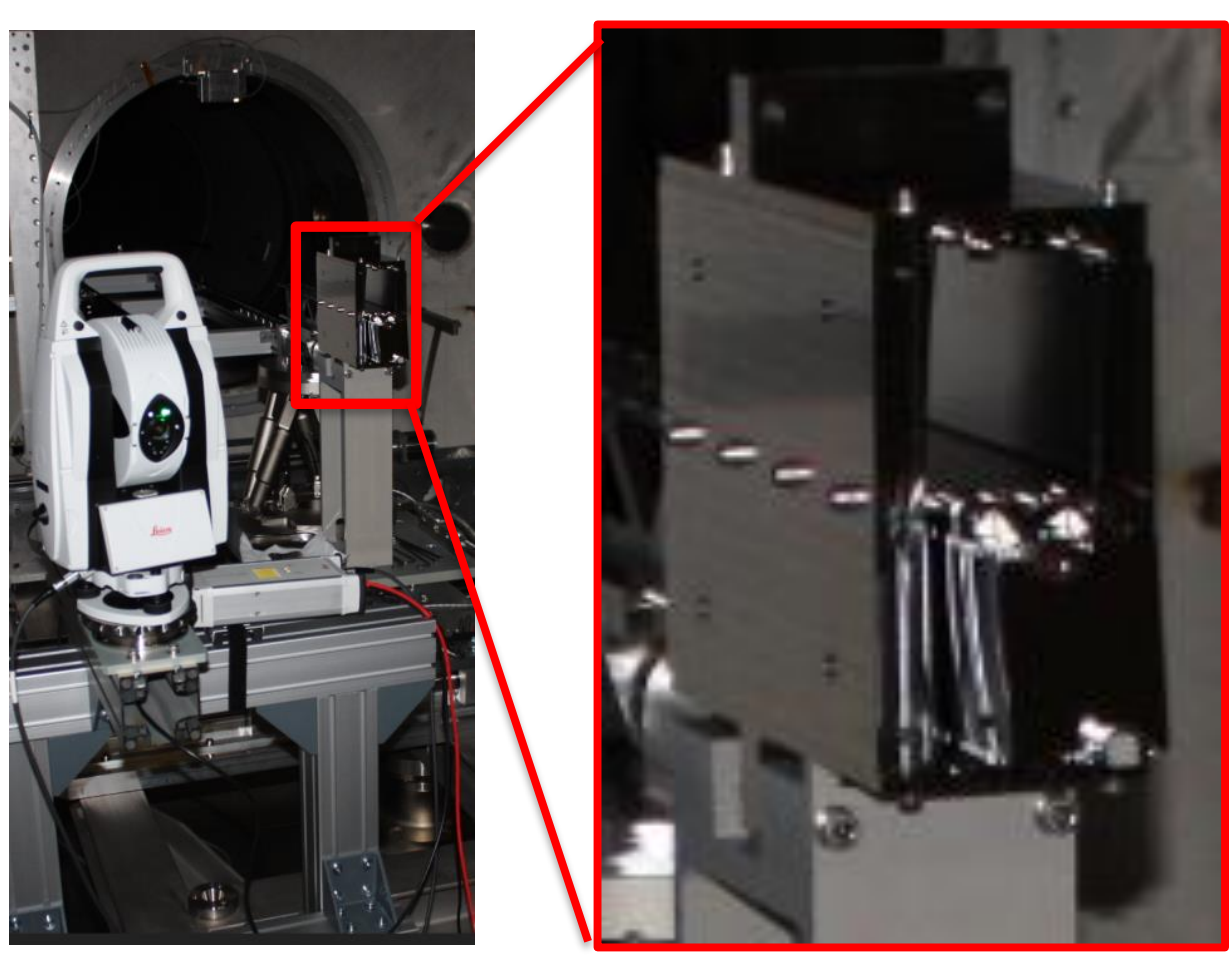




\section{ATHENA: Optics Calibration at PANTER}

\section{General considerations}

- Testing the X-ray optics with a finite source distance introduces:

- Focal plane shift $\leftarrow$ thin lense equation

- Collecting area losses

- PSF aberrations

- The magnitude of the errors are dependant on

$\rightarrow$ beam divergence $\rightarrow$ thus on the beam length and diameter 


\section{ATHENA: Optics Calibration at PANTER}

Mirror Performance verification

- Using a source at „quasi“ infinity is required to check:

- the Alignment of integrated modules in mirror structure $\rightarrow$ to achieve 5 arcsec angular resolution

- that the focal length error is $<1 \mathrm{~mm}$

- the collecting area error is $<10 \%$

- That the aberrations a $<1$ arcsec

- A full aperture calibration requires X-ray beam line with

- Length $>800 \mathrm{~m}$ for illuminating $>90 \%$ of the Mirror

- Tube Diameter expanding from $1.0 \mathrm{~m}$ at X-ray source to $3.2 \mathrm{~m}$ at the Test chamber

$\rightarrow$ To meet the specified accuracy 


\section{ATHENA: Optics Calibration at PANTER}

- If no large new facility is available for calibration Sectorwise illumination and PSF and EA stitching becomes necessary.

- At the existing PANTER with a source distance $123 \mathrm{~m}$ a maximum of 10 modules could be illuminated simultaneously to meet the accuracy requirements thus about 100 subapertures would have to be measured.

- As the source distance increases the less subapertures are need due to the smaller beam divergence.

- But the whole process is dominated by the errors introduced by the precise knowledge of the relative positions of the mirror with respect to the test camera (use of laser trackers required)

- The more sectors to be measured the longer the time required for Mirror calibration 


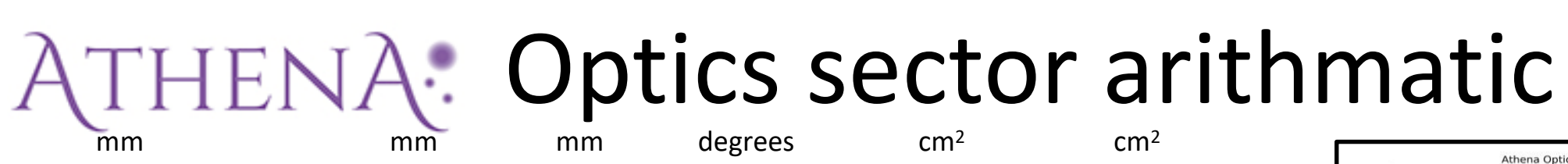

\# sectors

12

$R$ in

250

\# rows

Delta R

8250

250

$19 \quad 1235$

R out

1485

1485

Angle

Area of a sector

Total Area

$19 \quad 1235$

1235

$\begin{array}{ll}6 & 250\end{array}$

$250 \quad 19 \quad 1235$

1485

\# sectors

6

12

$\mathrm{R}$ in

\# rows

Delta R

1485

30

45

1833

22000

2750

22000

$60 \quad 3667 \quad 22000$

360

22000

R out

640

390

455

1095

640

7

390

1485

1235

30

\# sectors

6

12

18

36

\# sectors

12

24

24

$R$ in

\# rows

Delta $\mathrm{R}$

$\mathrm{R}$ out

390

455

390

640

640

1095

7

1095

1235

$R$ in

\# rows

Delta R

1485

Angle

Area of a sector

594

675

861

2130

Angle

60

Area of a sector

594

675

574

1843

R out Angle

455

705

1160

705

455

325

1485

30

Area of a sector

372

363

368

1103

15

1235

15

otal Area

3564

8105

10331

22000

Total Area

3564

8105

10331

22000

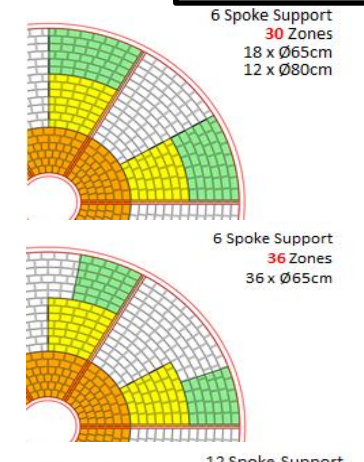

Total Area

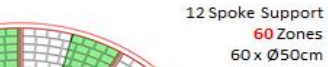

4461

8713

8826

22000
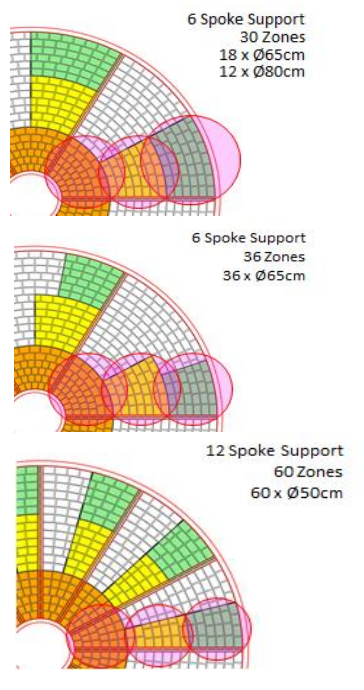

Vadim Burwitz (MPE) ２018-10-10 ICSO Conf. Chania, Crete, Greece 
New X-ray Test Facility for ATHENA:

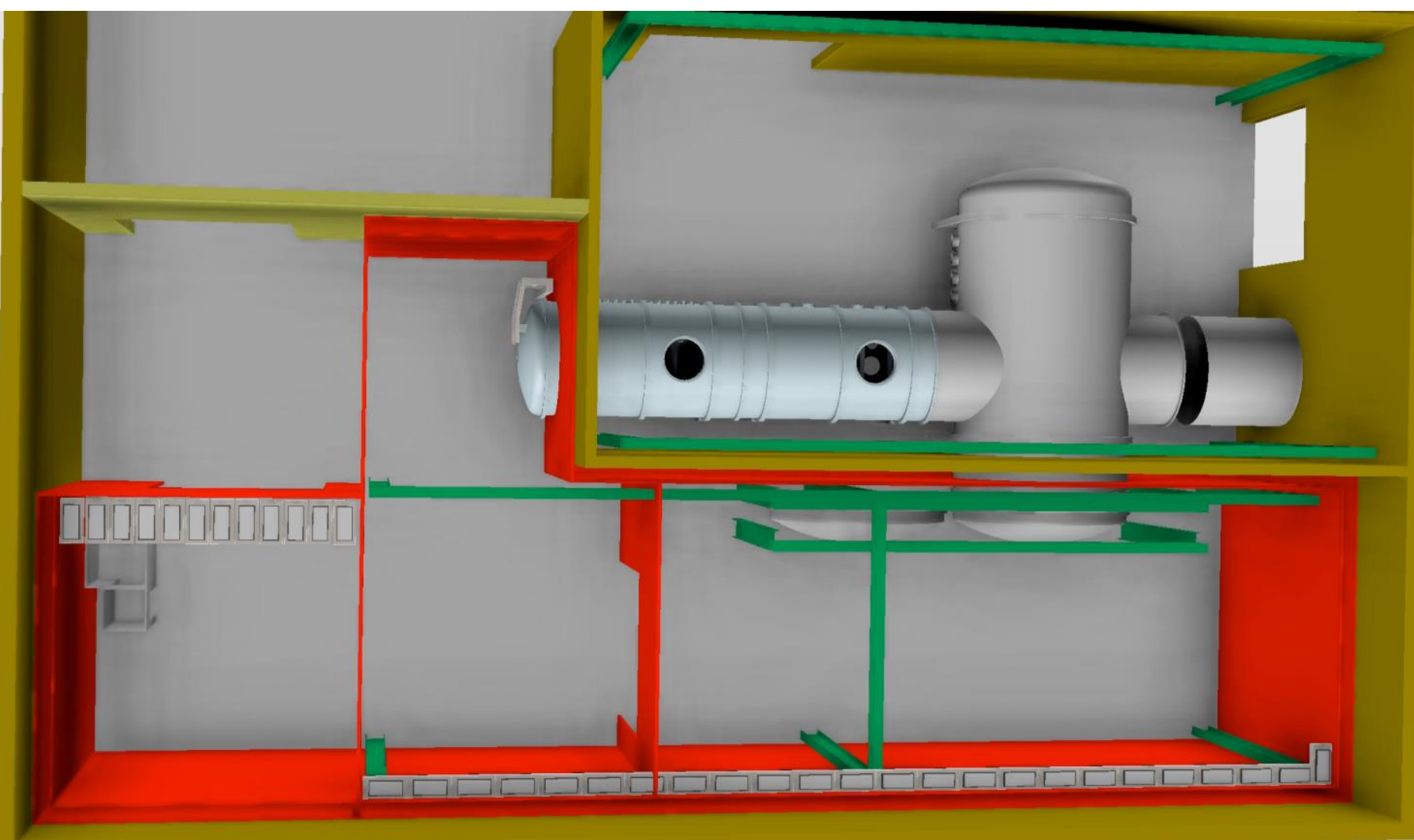




\section{New X-ray Test Facility for ATHENA:}

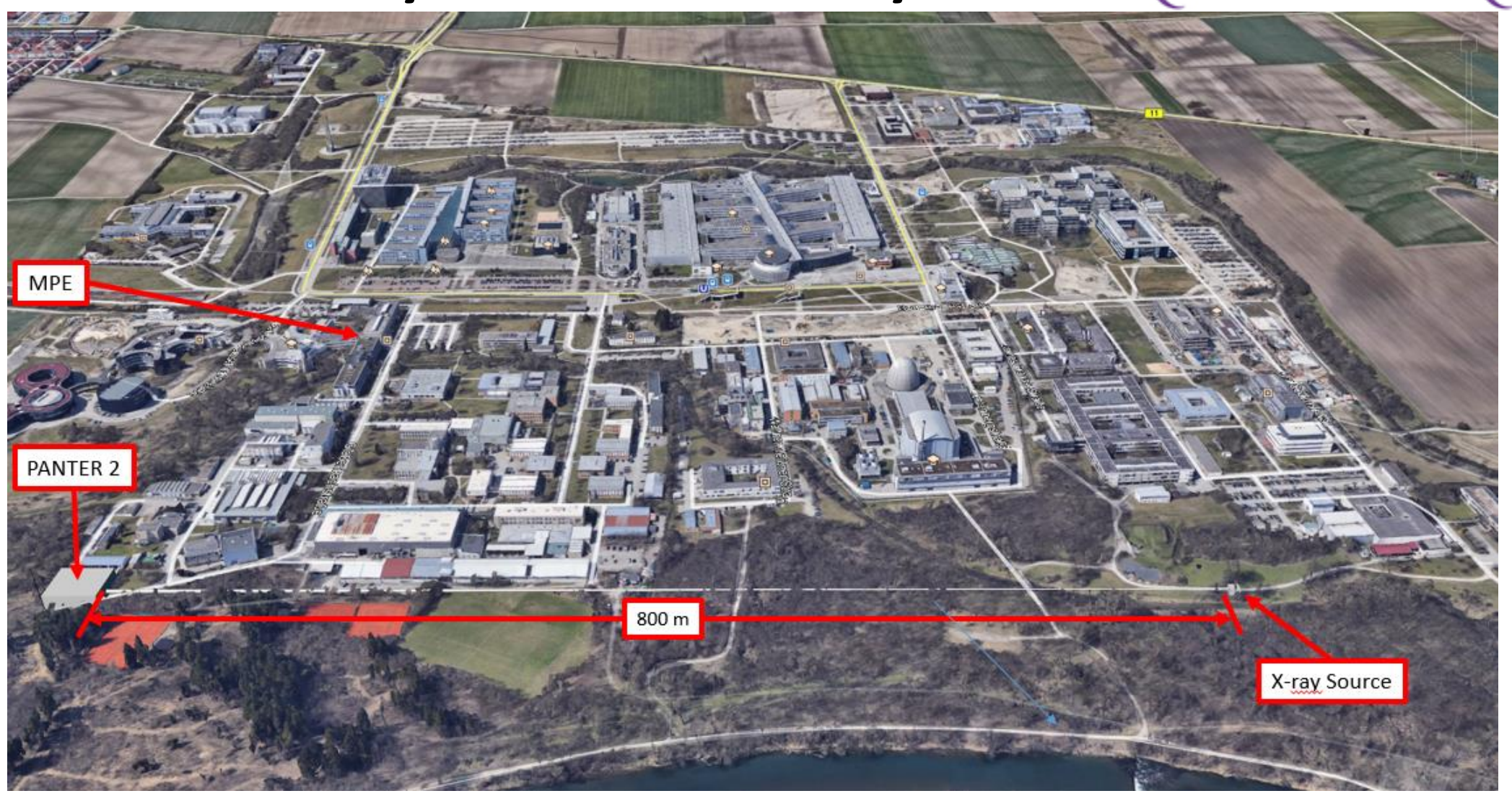




\section{Summary}

- Many upgrades to the current facility have been done

$\rightarrow$ to acommodate the $f=12 \mathrm{~m}$ optics and AIT petals

$\rightarrow$ to test optics on XOU level without glueing

- The Arcus petal size just compatible with PANTER facility

$\rightarrow$ all tests needed for the flight models are possible

- For ATHENA final trade studies off full- vs. sectorwise-

illumination of the fully populated mirror is ongoing.

$\rightarrow$ to decide what type of facility will be needed 
END 